\title{
Interactive effect of genetic susceptibility with height, body mass index, and hormone replacement therapy on the risk of breast cancer
}

\author{
Sophia Harlid ${ }^{1,2}$, Salma Butt ${ }^{3}$, Malin IL Ivarsson 1,2,4, Jorunn Erla Eyfjörd ${ }^{5}$, Per Lenner ${ }^{6}$, Jonas Manjer ${ }^{3}$,
} Joakim Dillner ${ }^{1,8}$ and Joyce Carlson $2,7^{*}$

\begin{abstract}
Background: Breast cancer today has many established risk factors, both genetic and environmental, but these risk factors by themselves explain only part of the total cancer incidence. We have investigated potential interactions between certain known genetic and phenotypic risk factors, specifically nine single nucleotide polymorphisms (SNPs) and height, body mass index (BMI) and hormone replacement therapy (HRT).

Methods: We analyzed samples from three different study populations: two prospectively followed Swedish cohorts and one Icelandic case-control study. Totally 2884 invasive breast cancer cases and 4508 controls were analysed in the study. Genotypes were determined using Mass spectrometry-Maldi-TOF and phenotypic variables were derived from measurements and/or questionnaires. Odds Ratios and 95\% confidence intervals were calculated using unconditional logistic regression with the inclusion of an interaction term in the logistic regression model.

Results: One SNP (rs851987 in ESR1) tended to interact with height, with an increasingly protective effect of the major allele in taller women ( $p=0.007$ ) and rs13281615 (on 8q24) tended to confer risk only in non users of HRT ( $p$-for interaction $=0.03$ ). There were no significant interactions after correction for multiple testing.

Conclusions: We conclude that much larger sample sets would be necessary to demonstrate interactions between low-risk genetic polymorphisms and the phenotypic variables height, BMI and HRT on the risk for breast cancer. However the present hypothesis-generating study has identified tendencies that would be of interest to evaluate for gene-environment interactions in independent materials.
\end{abstract}

\section{Background}

Genome wide association studies (GWAS), have discovered several new genetic polymorphisms affecting breast cancer risk [1-3]. Even though these individual riskfactors each confer quite small increases in risk, a positive association is seen between the number of risk alleles carried and risk for breast cancer $[4,5]$.

The phenotypic variables height, body mass index (BMI) and use of hormone replacement therapy (HRT) reflect to varying degrees genetic background and environmental exposure. Both height and BMI have previously been shown to associate with breast cancer

\footnotetext{
* Correspondence: joyce.carlson@med.lu.se

${ }^{2}$ Department of Clinical Chemistry, Lund University, Malmö, Sweden

'Labmedicin Skåne, Clinical Chemistry in Lund, Lund, Sweden Full list of author information is available at the end of the article
}

[6,7]. Increase in height has been shown to yield a proportional increase in breast cancer risk and obese women have a greater risk to contract postmenopausal breast cancer. Increased risk is also established for users of HRT [7], which has been speculated to interact with low-risk polymorphisms in the FGFR2 gene $[8,9]$.

Although there have been investigations on geneenvironment interactions in breast cancer [10], this area remains to a large extent unexplored.

The aim of this study was to investigate if height, BMI and HRT modify the genetic predisposition to breast cancer conferred by reported low-risk polymorphisms. For this purpose we had access to two well defined Swedish population based cohorts as well as an Icelandic hospital based case control study, altogether 7738 samples (3016 cases and 4722 controls).

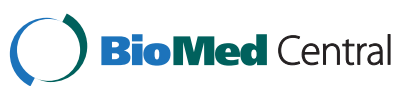




\section{Methods}

\section{Study populations}

The samples originate from two Swedish independent population based cohorts; the Malmö Diet and Cancer Study (MDCS) from southern Sweden and the North Sweden Health and Disease Study (NSHDS), together comprising 2410 incident cases and 3829 controls. The third sample collection was an Icelandic population-based case control study including 866 cases and 948 controls. Written informed consent was retrieved from all women prior to donating their samples. All cohorts have been described previously [11] and are briefly presented below.

\section{MDCS}

The Malmö Diet and Cancer Study (MDCS) is a prospective cohort study initiated in 1991. Totally it comprises 17035 female residents of Malmö Sweden recruited between 1991 and 1996 [12,13]. By linkage to the national cancer registry until $31^{\text {st }}$ of December 2007, 730 incident cases of invasive breast cancer were identified among MDCS participants. They were matched to 1460 controls from the same cohort according to sex, age (+/ 6 months), and date of sampling at baseline (+/- 2 months). Median age at breast cancer diagnosis was 65 years (range 45-84). Thirty-three cases and 65 controls were $\leq 50$ years of age at time of diagnosis.

The MDCS and the present analyses were approved by the Ethical Committee at Lund University (LU 51-90, Dnr 652/2005 and Dnr 2009/682).

\section{NSHDS}

The Northern Sweden Health and Disease Study (NSHDS) include the Västerbotten Intervention Program (VIP), and the Mammography Screening Program (MSP), initiated in 1985 and 1995 respectively. Participants in the VIP are screened at 40, 50 and 60 years of age and mammography screening and blood sampling is performed among women between 50 and 69 years of age [14]. Through linkage with the cancer register up to December $31^{\text {st }}, 2008,1680$ prospective cases of invasive breast cancer (median age 56 years, range 27-95) were identified. They were matched to 2314 controls by sex, age (+/ -6 months), and date of sampling at baseline (+/- 2 months), (474 cases and 606 controls $\leq 50$ years of age). Information on HRT use was available for 1420 of these cases.

The NSHDS and the present analyses were approved by the Ethical Committee at Umeå University (Dnr: 2010-147-132 and 07-141).

\section{ICELAND}

The Icelandic samples were collected between 1998 and 2006 and represent 45-77\% of all Icelandic women with invasive breast cancer diagnosed between 1957 and
2007. The rate of participation varied somewhat depending on the year of diagnosis and was highest between 1999 and 2003 (77\%). Unmatched controls were collected between 2000 and 2004, either from women who participated in the population-based cervical or breast cancer screening program and found free of breast cancer or from older women in retirement homes who had not been diagnosed with breast cancer, to generally reflect the ages of the cases. By linkage to the Icelandic cancer registry in 2008 we identified cases diagnosed before $31^{\text {st }}$ of December 2007 . Totally 866 cases (median age 55 years, range $22-98,314 \leq 50$ years) and 948 controls (median age 58 years, range $25-102,256 \leq 50$ years) had DNA available and were eligible to us.

The use of these samples was approved by the data protection law (200605037), and the Icelandic Science Ethics Committee (VSNb2006050001/03-16 and VSNb2005070008/ 03-16).

\section{Data collection}

Participants in both Swedish cohorts completed a questionnaire providing information about current medication at the time of recruitment. Participants in the NSHDS also provided information about height and weight while a trained nurse at the study centre measured height and weight, for participants in MDCS [15].

The Icelandic women answered questions about height, weight and HRT use when they attended the Detection Cancer Clinic (breast cancer mammography or cervical screening) at the Icelandic Cancer Society. The women answered questions at least every tenth year and the most recent answers were used in the study. For the Icelandic cases only data collected prior to breast cancer diagnosis was used. BMI for all participants was calculated as $\mathrm{kg} / \mathrm{m}^{2}$.

\section{SNP selection}

All loci identified by GWAS to be associated with breast cancer and published before June $31^{\text {st }} 2007$ were initially included in the study [1-3]. Individual SNPs were selected from the publications by Easton et al. and Stacey et al. This primary selection included 10 SNPs, as well as one SNP in CASP8 identified using the candidate gene approach [16]. Two SNPs selected from our own candidate CpG SNP study [11] were also included making a total of 13 SNPs (Figure 1).

\section{Assay design and genotyping}

Eleven SNPs, combined by the SEQUENOM MassARRAY ${ }^{\circledR}$ Designer software in a single multiplex assay were analyzed on a MALDI-TOF mass spectrometer (SEQUENOM MassArray) using standard iPLEX reagents and protocol (SEQUENOM) and $10 \mathrm{ng}$ DNA as PCR template. Primer sets were from Metabion (Martinsried, Germany). 


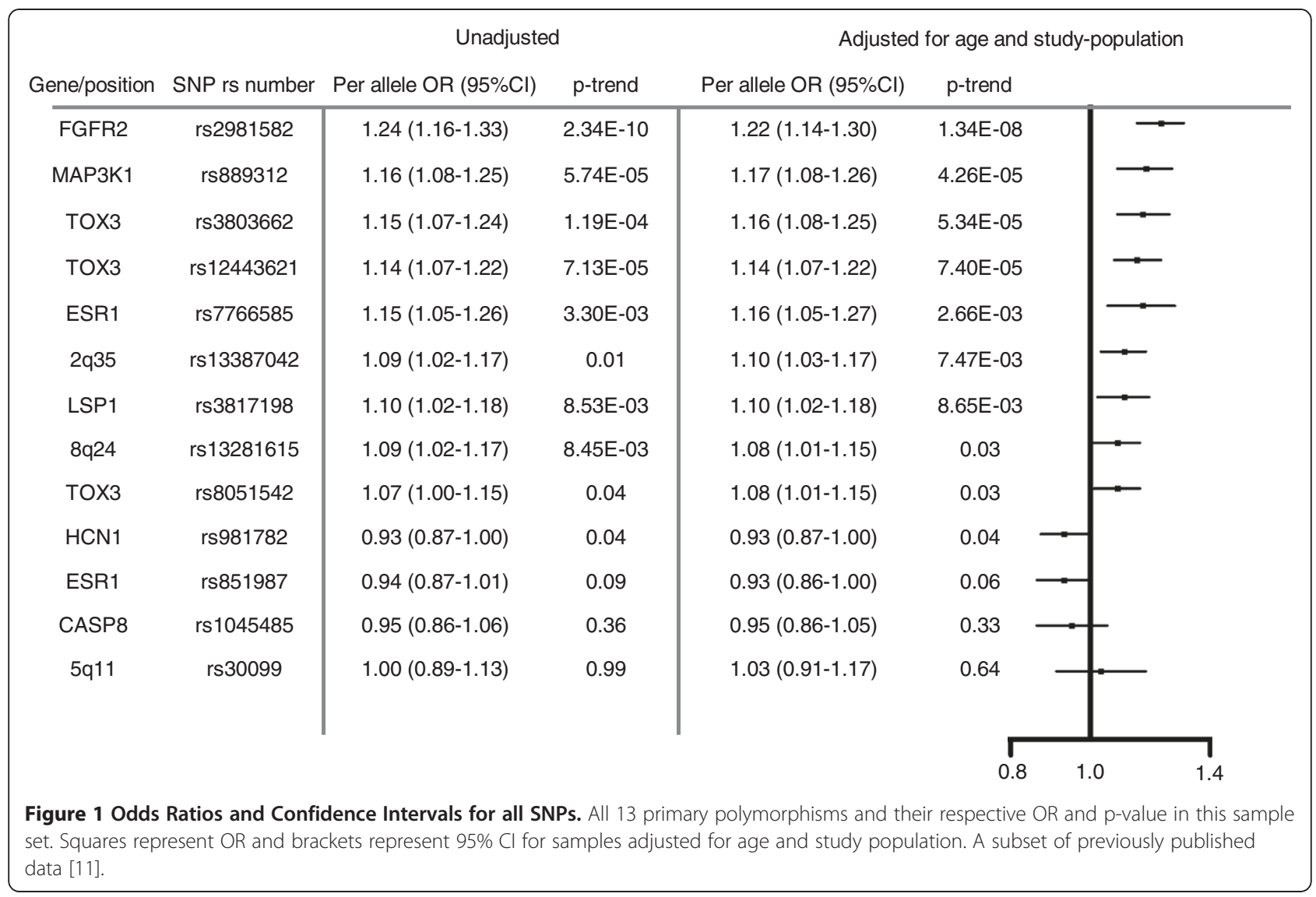

SNPs rs2981582 and rs1045485 were analyzed by a separate TaqMan ${ }^{\circledR}$ "assay by design" genotyping assay on a $7900 \mathrm{HT}$ instrument, using Master mix No UNG from Applied Biosystems according to the manufacturer's instructions. Reaction mixtures $(6 \mu \mathrm{L})$ containing 2 ng of DNA template, primers (rs2981582 forward primer 5' -CAG CAC TCA TCG CCA CTT AAT G-3', reverse primer 5'-GAC ACC ACT CGG ACT GCT-3', and probes 5'-VIC-TCT CCG CAA ACA GG-MGB-3' and 5'-FAM-CTC TCC ACA AAC AGG-MGB-3') (rs1045485 forward primer 5'-ACC ACG ACC TTT GAA GAG CTT -3', reverse primer 5'-ACT GTG GTC CAT GAG TTG GTA GAT-3', and probes 5'-VICCCC CAC GAT GAC TG-MGB-3' and 5'-FAM-CCC CAC CAT GAC TG-MGB-3') were subjected to two minutes at $50^{\circ} \mathrm{C}$ and ten minutes at $95^{\circ} \mathrm{C}$, followed by 50 PCR cycles of $95^{\circ} \mathrm{C}$ for 15 seconds and $60^{\circ} \mathrm{C}$ for one minute.

Three percent of the samples from NSHDS and five percent of the Icelandic samples were included as blinded duplicates for quality control purposes.

\section{Statistical analysis}

Individual samples producing results in $<80 \%$ of the assays were excluded prior to statistical analyses to eliminate samples with low-quality DNA. Genotype data from control samples were tested for consistency with Hardy-Weinberg equilibrium (HWE) using a $\chi^{2} \mathrm{p}$-value cutpoint of 0.001 . Unconditional logistic regression was used to measure the independent association between each genotype and breast cancer, with Odds ratios and 95\% confidence intervals (CI) estimated for each genotype. Per allele OR (p-trend) was calculated using 0, 1 or 2 copies of the minor allele (a) as a continuous variable. OR and 95\% CI were calculated between each phenotypic variable (Height, BMI and HRT) and risk for breast cancer, these results were also age adjusted. Data was then stratified into tertiles according to height $(<162 \mathrm{~cm}$, 162-166 am and $>166 \mathrm{~cm}$ ), and into subcategories of BMI according to the WHO guidelines (Normal weight: 18.5-25, Overweight: $25-30$ and Obese $>30$ ). For HRT subjects data was stratified according to reported "non use" and "current use". The current users were further divided into users of only estrogen or combined hormones. OR and 95\%CI were calculated for each variable (Height, BMI and HRT) and risk for breast cancer.

A p-value for interaction was estimated for each pair of genotype/phenotype and a value of less than 0.05 was considered statistically significant. As adjustment for multiple comparisons this value was divided by the number 
of interaction analyses, according to Bonferroni, (8 SNPs x $3=24$ ) and the new significance threshold was 0.002 . All results were adjusted for age and study population.

\section{Results}

Of the initial 7738 samples selected for the project 7392 (95.5\%) were successfully retrieved and genotyped for $\geq$ $80 \%$ of the SNPs. All SNPs had a genotyping success rate $>94 \%$, with an average of $98.0 \%$. Results of all 200 analyses performed on duplicate samples were in $100 \%$ concordance.

Per allele OR for each independent SNP is presented in Figure 1. Ten of the SNPs were significant $(\mathrm{p}<0.05)$ in our material with rs2981852 (FGFR2), rs889312 (MAP3K1) and rs3803662 (TOX3) exhibiting the highest ORs. Two of the SNPs had p-values >0.1 (rs1045485 [CASP8] and rs30099 [5q11]) and were excluded from further analysis.

Three of the SNPs in TOX3 (rs3803662, rs12443621 and rs8051542) exhibited linkage (results not shown) as has previously been reported [1,4]. Rs12443621 and rs80515442 were therefore excluded from further analysis.

Independent analysis of risk association with each phenotypic variable (height, BMI, HRT) within the entire study population revealed a significantly increased risk of breast cancer for individuals $>162 \mathrm{~cm}$ compared to shorter women, this association was weakened following age adjustment. No statistical significant correlation between BMI and risk for breast cancer was found in this population. For current use vs. non-use of HRT, a significantly increased risk was seen for users, OR (95\% CI) 1.24 (1.08-1.42), which remained after adjustment for age (Table 1).

\section{Stratified analysis and interactions}

After stratification by height (as described in materials and methods), one SNP (rs851987) in ESR1 had a p-interaction $=0.007$ with height, with an increasingly protective effect of the major allele in taller women, but it did not pass the threshold for multiple comparisons $(\mathrm{p}=0.002)$ (Table 2).

None of the SNPs showed any tendencies towards significant interactions after stratification according to BMI (Table 3).

Following stratification of genotypes according to reported current use or non-use of hormone replacement therapy, rs13281615 (8q24) was significant only in non users of HRT with a p-for interaction of 0.03 , indicating borderline significance (Table 4).

\section{Discussion}

In this study we have explored interactions between reported genetic risk factors for breast cancer and the three additional established risk factors; height, BMI and HRT in 2884 cases and 4508 controls. The strongest tendency for interaction found was that between height and rs851987 in ESR1, although it did not pass the threshold for multiple comparisons. Taller women carrying the T-allele appeared to have reduced breast cancer risk ( $\mathrm{p}$ for interaction $=0.007)$ (Table 2). Rs851987 was described

Table 1 Environmental risk factors (MDCS, NSHDS and ICELAND)

\begin{tabular}{|c|c|c|c|c|c|c|c|c|c|}
\hline \multicolumn{2}{|c|}{ Risk factor Categories } & \multicolumn{2}{|c|}{ MDCS } & \multicolumn{2}{|c|}{ NSHDS } & \multicolumn{2}{|c|}{ ICELAND } & \multicolumn{2}{|c|}{ TOTAL } \\
\hline & & Count & $\mathrm{OR}^{*}$ & Count & $\mathrm{OR}^{*}$ & Count & $\mathrm{OR}^{*}$ & Count & $\mathrm{OR}^{*}$ \\
\hline & & Case/Control & $(95 \% \mathrm{Cl})$ & Case/Control & $(95 \% \mathrm{Cl})$ & Case/Control & $(95 \% \mathrm{Cl})$ & Case/Control & $(95 \% \mathrm{Cl})$ \\
\hline \multicolumn{10}{|c|}{ All individuals } \\
\hline \multirow[t]{3}{*}{$\mathrm{BMI}$} & Normal Weight & $334 / 695$ & 1 & $637 / 997$ & 1 & $361 / 326$ & 1 & $1332 / 2018$ & 1 \\
\hline & Over weight & $237 / 450$ & $1.26(1.02-1.56)$ & $439 / 701$ & $0.99(0.84-1.15)$ & $241 / 312$ & $0.76(0.60-0.96)$ & $917 / 1463$ & $1.00(0.90-1.12)$ \\
\hline & Obese & $95 / 194$ & $1.25(0.94-1.66)$ & $167 / 286$ & $0.91(0.74-1.14)$ & $112 / 139$ & $0.79(0.59-1.07)$ & $374 / 619$ & $0.97(0.84-1.13)$ \\
\hline \multirow[t]{3}{*}{ Height } & $<162 \mathrm{~cm}$ & $207 / 471$ & 1 & $398 / 722$ & 1 & $171 / 173$ & 1 & $776 / 1366$ & 1 \\
\hline & $162-166 \mathrm{~cm}$ & $233 / 443$ & $1.03(0.81-1.30)$ & $445 / 657$ & $1.21(1.02-1.44)$ & 289/307 & $0.92(0.70-1.20)$ & $967 / 1407$ & $1.15(1.02-1.30)$ \\
\hline & $>166 \mathrm{~cm}$ & $233 / 448$ & $0.93(0.73-1.18)$ & $436 / 640$ & $1.21(1.01-1.44)$ & $354 / 414$ & $0.78(0.60-1.02)$ & $1023 / 1502$ & $1.09(0.97-1.23)$ \\
\hline \multicolumn{10}{|c|}{$>50$ years } \\
\hline \multirow[t]{2}{*}{ HRT } & Non users & $341 / 806$ & 1 & $777 / 1320$ & 1 & $66 / 286$ & 1 & $1184 / 2412$ & 1 \\
\hline & Current users & $181 / 256$ & $1.26(0.99-1.61)$ & $186 / 295$ & 1.09 (0.89-1.34) & $105 / 225$ & $2.06(1.45-2.95)$ & $424 / 776$ & $1.22(1.07-1.39)$ \\
\hline \multirow[t]{3}{*}{$\overline{\mathrm{BMI}}$} & Normal Weight & $322 / 694$ & 1 & $400 / 682$ & 1 & $189 / 217$ & 1 & $911 / 1593$ & 1 \\
\hline & Over weight & $232 / 449$ & $1.26(1.02-1.57)$ & $320 / 504$ & $1.09(0.91-1.32)$ & $188 / 255$ & $0.85(0.65-1.12)$ & $740 / 1208$ & $1.12(0.99-1.26)$ \\
\hline & Obese & $95 / 194$ & $1.26(0.94-1.68)$ & $130 / 205$ & $1.10(0.85-1.41)$ & $86 / 109$ & $0.91(0.65-1.29)$ & $311 / 508$ & $1.13(0.96-1.34)$ \\
\hline \multirow[t]{3}{*}{ Height } & $<162 \mathrm{~cm}$ & $205 / 471$ & 1 & $283 / 534$ & 1 & $125 / 136$ & 1 & $613 / 1141$ & 1 \\
\hline & $162-166 \mathrm{~cm}$ & $224 / 442$ & $1.02(0.81-1.29)$ & $311 / 458$ & $1.26(1.03-1.55)$ & $198 / 244$ & $0.88(0.64-1.19)$ & $733 / 1144$ & $1.13(0.99-1.30)$ \\
\hline & $>166 \mathrm{~cm}$ & $227 / 447$ & $0.94(0.74-1.19)$ & $279 / 421$ & $1.22(0.99-1.51)$ & $199 / 274$ & $0.78(0.57-1.06)$ & $705 / 1142$ & $1.05(0.92-1.21)$ \\
\hline
\end{tabular}

*Adjusted for age. 
Table 2 OR adjusted for Age and Study Population, stratified by Height

\begin{tabular}{|c|c|c|c|c|c|c|c|c|c|c|}
\hline \multirow[t]{3}{*}{ SNP rs nr } & \multirow[t]{3}{*}{ Genotype } & \multirow[t]{3}{*}{$\begin{array}{l}p \text {-value for } \\
\text { interaction }\end{array}$} & \multicolumn{2}{|c|}{ All individuals } & \multicolumn{2}{|c|}{ Height $<162.0 \mathrm{~cm}$} & \multirow{2}{*}{\multicolumn{2}{|c|}{$\begin{array}{l}\text { Height } 162.0-166.0 \mathrm{~cm} \\
\text { case/control: } 967 / 1407\end{array}$}} & \multirow{2}{*}{\multicolumn{2}{|c|}{$\begin{array}{c}\text { Height }>166.0 \mathrm{~cm} \\
\text { case/control: } 1023 / 1502\end{array}$}} \\
\hline & & & \multicolumn{2}{|c|}{ case/contr: $2869 / 4480$} & \multicolumn{2}{|c|}{ case/control: $776 / 1366$} & & & & \\
\hline & & & OR 95\%Cl & p-trend & OR 95\%Cl & p-trend & OR $95 \% \mathrm{Cl}$ & p-trend & OR $95 \% \mathrm{Cl}$ & p-trend \\
\hline \multirow[t]{4}{*}{ FGFR2 2981582} & $\mathrm{AA}$ & & 1 & & 1 & & 1 & & 1 & \\
\hline & $\mathrm{Aa}$ & & $1.23(1.10-1.37)$ & & $1.35(1.10-1.66)$ & & $1.33(1.10-1.61)$ & & $1.07(0.89-1.28)$ & \\
\hline & aа & & $1.48(1.29-1.69)$ & & $1.57(1.21-2.02)$ & & $1.56(1.22-1.99)$ & & $1.32(1.04-1.67)$ & \\
\hline & Per Allele & 0,41 & $1.22(1.14-1.30)$ & $1.34 \mathrm{E}-08$ & $1.26(1.11-1.43)$ & $2.86 \mathrm{E}-04$ & $1.26(1.12-1.42)$ & $1.65 \mathrm{E}-04$ & $1.14(1.01-1.28)$ & 0.03 \\
\hline \multirow[t]{4}{*}{ TNR09 3803662} & $\mathrm{AA}$ & & 1 & & 1 & & 1 & & 1 & \\
\hline & $\mathrm{Aa}$ & & $1.14(1.03-1.26)$ & & $1.09(0.90-1.32)$ & & $1.23(1.03-1.47)$ & & $1.15(0.97-1.36)$ & \\
\hline & aа & & $1.39(1.17-1.67)$ & & $1.37(1.00-1.87)$ & & $1.37(1.00-1.89)$ & & $1.42(1.03-1.95)$ & \\
\hline & Per Allele & 0,91 & $1.16(1.08-1.25)$ & 5.34E-05 & $1.14(1.00-1.31)$ & 0.06 & $1.20(1.05-1.37)$ & $6.78 \mathrm{E}-03$ & $1.17(1.03-1.33)$ & 0.02 \\
\hline \multirow[t]{4}{*}{ MAP3K1 889312} & AA & & 1 & & 1 & & 1 & & 1 & \\
\hline & $\mathrm{Aa}$ & & $1.21(1.09-1.34)$ & & $1.29(1.06-1.55)$ & & $1.14(0.95-1.36)$ & & $1.18(1.00-1.40)$ & \\
\hline & aа & & $1.29(1.09-1.54)$ & & $1.31(0.95-1.81)$ & & $1.05(0.76-1.45)$ & & $1.53(1.14-2.06)$ & \\
\hline & Per Allele & 0,45 & $1.17(1.08-1.26)$ & 4.26E-05 & $1.20(1.04-1.37)$ & 9.92E-03 & $1.07(0.94-1.22)$ & 0.29 & $1.22(1.07-1.38)$ & 2.20E-03 \\
\hline \multirow[t]{4}{*}{$8 q 2413281615$} & $\mathrm{AA}$ & & 1 & & 1 & & 1 & & 1 & \\
\hline & $\mathrm{Aa}$ & & $1.12(1.00-1.24)$ & & $1.21(0.98-1.48)$ & & $1.07(0.89-1.29)$ & & $1.12(0.93-1.34)$ & \\
\hline & aа & & $1.15(1.00-1.32)$ & & $1.24(0.96-1.60)$ & & $1.17(0.92-1.49)$ & & 1.09 (0.87-1.38) & \\
\hline & Per Allele & 0,90 & $1.08(1.01-1.15)$ & 0.03 & $1.12(0.99-1.27)$ & 0.07 & $1.08(0.96-1.22)$ & 0.20 & $1.06(0.94-1.18)$ & 0.35 \\
\hline \multirow[t]{4}{*}{ LSP1 3817198} & $\mathrm{AA}$ & & 1 & & 1 & & 1 & & 1 & \\
\hline & $\mathrm{Aa}$ & & $1.12(1.02-1.24)$ & & $1.19(0.99-1.45)$ & & $1.08(0.90-1.28)$ & & $1.08(0.91-1.28)$ & \\
\hline & aa & & $1.19(1.00-1.40)$ & & $1.50(1.11-2.02)$ & & $1.14(0.84-1.55)$ & & $1.04(0.79-1.38)$ & \\
\hline & Per Allele & 0,48 & $1.10(1.02-1.18)$ & $8.65 E-03$ & 1.21 (1.06-1.39) & 4.41E-03 & $1.07(0.94-1.22)$ & 0.29 & $1.04(0.92-1.18)$ & 0.50 \\
\hline \multirow[t]{4}{*}{$2 q 35$} & $\mathrm{AA}$ & & 1 & & 1 & & 1 & & 1 & \\
\hline & $\mathrm{Aa}$ & & $1.05(0.93-1.19)$ & & $0.90(0.72-1.14)$ & & $1.21(0.98-1.50)$ & & $0.97(0.79-1.19)$ & \\
\hline & aa & & $1.20(1.05-1.37)$ & & $1.09(0.85-1.40)$ & & $1.28(1.01-1.63)$ & & $1.21(0.96-1.52)$ & \\
\hline & Per Allele & 0,38 & $1.10(1.03-1.17)$ & 7.47E-03 & $1.05(0.93-1.19)$ & 0.43 & $1.13(1.00-1.27)$ & 0.04 & $1.10(0.98-1.23)$ & 0.11 \\
\hline \multirow[t]{4}{*}{ ESR1 7766585} & AA & & 1 & & 1 & & 1 & & 1 & \\
\hline & $\mathrm{Aa}$ & & $1.23(1.10-1.37)$ & & $1.12(0.90-1.38)$ & & $1.26(1.03-1.53)$ & & $1.25(1.04-1.50)$ & \\
\hline & aа & & $1.02(0.74-1.41)$ & & $0.83(0.43-1.58)$ & & $1.21(0.69-2.13)$ & & $0.98(0.57-1.66)$ & \\
\hline & Per Allele & 0,83 & $1.16(1.05-1.27)$ & 2.66E-03 & $1.05(0.88-1.26)$ & 0.60 & $1.20(1.02-1.42)$ & 0.03 & $1.16(0.99-1.36)$ & 0.06 \\
\hline \multirow[t]{4}{*}{ HCN1 981782} & $\mathrm{AA}$ & & 1 & & 1 & & 1 & & 1 & \\
\hline & $\mathrm{Aa}$ & & $0.97(0.87-1.09)$ & & $0.92(0.74-1.14)$ & & $1.03(0.84-1.26)$ & & $1.00(0.82-1.21)$ & \\
\hline & aа & & $0.86(0.75-0.99)$ & & $0.91(0.70-1.18)$ & & $0.96(0.75-1.22)$ & & $0.77(0.61-0.98)$ & \\
\hline & Per Allele & 0,51 & $0.93(0.87-1.00)$ & 0.04 & $0.95(0.83-1.08)$ & 0.44 & $0.98(0.87-1.11)$ & 0.73 & $0.88(0.78-0.99)$ & 0.04 \\
\hline \multirow[t]{4}{*}{ ESR1 851987} & $\mathrm{AA}$ & & 1 & & 1 & & 1 & & 1 & \\
\hline & $\mathrm{Aa}$ & & $0.91(0.82-1.02)$ & & $0.96(0.78-1.17)$ & & $0.92(0.76-1.11)$ & & $0.87(0.73-1.05)$ & \\
\hline & aа & & $0.88(0.74-1.05)$ & & $1.44(1.06-1.95)$ & & $0.69(0.50-0.96)$ & & $0.71(0.52-0.98)$ & \\
\hline & Per Allele & $6.70 \mathrm{E}-03$ & $0.93(0.86-1.00)$ & 0.06 & $1.12(0.97-1.28)$ & 0.12 & $0.86(0.75-0.99)$ & 0.04 & $0.86(0.75-0.98)$ & 0.02 \\
\hline
\end{tabular}

None of the SNPs showed any tendencies towards significant interactions after stratification according to BMI (Table 3).

by Harlid et al. [11] and is situated in the far end of the extended promoter region of ESR1, about $3.7 \mathrm{~kb} \mathrm{5'}$ of exon $\mathrm{F}$. Exon $\mathrm{F}$ and its promoter were originally described by Thompson et al. [17] and have later been shown to affect the level of ESR1 expression in osteoblastic cells $[18,19]$. A potential association between ESR1 and height has been described in another study comprising adult males from two Swedish population cohorts [20]. Mutations in ESR1 have been reported to delay fusion of the epiphyseal plates at puberty [21], 
Table 3 OR adjusted for Age and Study Population, stratified by BMI

\begin{tabular}{|c|c|c|c|c|c|c|c|c|c|c|}
\hline \multirow[t]{4}{*}{ SNP rs nr } & \multirow[t]{4}{*}{ Genotype } & \multirow{4}{*}{$\begin{array}{l}p \text {-value for } \\
\text { interaction }\end{array}$} & \multicolumn{2}{|c|}{ All individuals } & \multirow{2}{*}{\multicolumn{2}{|c|}{$\begin{array}{l}\text { Normal Weigth } \\
\mathrm{BMI}=18.5-24.99\end{array}$}} & \multicolumn{2}{|c|}{ Over Weight } & \multicolumn{2}{|l|}{ Obese } \\
\hline & & & & & & & $\mathrm{BMI}=\mathbf{2 5 . 0 0}$ & -29.99 & $\mathrm{BMI} \geq 3 \mathrm{C}$ & \\
\hline & & & \multicolumn{2}{|c|}{ case/contr: $2869 / 4480$} & \multicolumn{2}{|c|}{ case/control: $1332 / 2018$} & \multicolumn{2}{|c|}{ case/control: $917 / 1463$} & \multicolumn{2}{|c|}{ case/control: $374 / 61 \mathrm{c}$} \\
\hline & & & OR $95 \% \mathrm{Cl}$ & p-trend & OR $95 \% \mathrm{Cl}$ & p-trend & OR $95 \% \mathrm{Cl}$ & $\mathrm{p}$-trend & OR $95 \% \mathrm{Cl}$ & p-trend \\
\hline \multirow[t]{4}{*}{ FGFR2 2981582} & $\mathrm{AA}$ & & 1 & & 1 & & 1 & & 1 & \\
\hline & $\mathrm{Aa}$ & & $1.23(1.10-1.37)$ & & $1.26(1.07-1.48)$ & & $1.18(0.97-1.42)$ & & $1.08(0.80-1.46)$ & \\
\hline & aа & & $1.48(1.29-1.69)$ & & $1.46(1.18-1.80)$ & & $1.57(1.24-1.99)$ & & $1.14(0.78-1.66)$ & \\
\hline & Per Allele & 0.59 & $1.22(1.14-1.30)$ & $1.34 \mathrm{E}-08$ & $1.21(1.10-1.35)$ & $2.03 \mathrm{E}-04$ & $1.25(1.11-1.40)$ & $2.20 \mathrm{E}-04$ & $1.07(0.89-1.29)$ & 0.49 \\
\hline \multirow[t]{4}{*}{ TNR09 3803662} & $\mathrm{AA}$ & & 1 & & 1 & & 1 & & 1 & \\
\hline & $\mathrm{Aa}$ & & $1.14(1.03-1.26)$ & & $1.16(1.00-1.35)$ & & $1.13(0.94-1.34)$ & & $1.27(0.97-1.67)$ & \\
\hline & aa & & $1.39(1.17-1.67)$ & & $1.44(1.10-1.89)$ & & $1.21(0.89-1.65)$ & & $1.72(1.07-2.78)$ & \\
\hline & Per Allele & 0.72 & $1.16(1.08-1.25)$ & $5.34 \mathrm{E}-05$ & $1.18(1.06-1.32)$ & $2.88 \mathrm{E}-03$ & $1.11(0.98-1.26)$ & 0.11 & $1.30(1.06-1.58)$ & 0.01 \\
\hline \multirow[t]{4}{*}{ MAP3K1 889312} & $A A$ & & 1 & & 1 & & 1 & & 1 & \\
\hline & $\mathrm{Aa}$ & & $1.21(1.09-1.34)$ & & $1.15(0.99-1.33)$ & & $1.33(1.11-1.58)$ & & $1.21(0.92-1.59)$ & \\
\hline & aа & & $1.29(1.09-1.54)$ & & $1.22(0.94-1.59)$ & & $1.63(1.20-2.22)$ & & $1.22(0.76-1.97)$ & \\
\hline & Per Allele & 0.47 & $1.17(1.08-1.26)$ & $4.26 \mathrm{E}-05$ & $1.12(1.01-1.25)$ & 0.04 & 1.30 (1.14-1.48) & 8.64E-05 & $1.15(0.94-1.40)$ & 0.18 \\
\hline \multirow[t]{4}{*}{$8 q 2413281615$} & $\mathrm{AA}$ & & 1 & & 1 & & 1 & & 1 & \\
\hline & $\mathrm{Aa}$ & & $1.12(1.00-1.24)$ & & $1.18(1.01-1.39)$ & & $1.04(0.86-1.25)$ & & $1.13(0.84-1.52)$ & \\
\hline & aа & & $1.15(1.00-1.32)$ & & $1.22(0.99-1.49)$ & & $1.14(0.90-1.45)$ & & $1.10(0.75-1.60)$ & \\
\hline & Per Allele & 0.82 & $1.08(1.01-1.15)$ & 0.03 & $1.11(1.01-1.23)$ & 0.04 & $1.06(0.95-1.20)$ & 0.30 & $1.06(0.88-1.27)$ & 0.54 \\
\hline \multirow[t]{4}{*}{ LSP1 3817198} & $\mathrm{AA}$ & & 1 & & 1 & & 1 & & 1 & \\
\hline & $\mathrm{Aa}$ & & $1.12(1.02-1.24)$ & & $1.16(0.99-1.34)$ & & $1.11(0.93-1.33)$ & & $0.94(0.72-1.24)$ & \\
\hline & aа & & $1.19(1.00-1.40)$ & & $1.41(1.10-1.81)$ & & $1.06(0.80-1.42)$ & & $0.83(0.50-1.38)$ & \\
\hline & Per Allele & 0.35 & $1.10(1.02-1.18)$ & $8.65 E-03$ & $1.18(1.06-1.31)$ & $3.22 \mathrm{E}-03$ & $1.06(0.93-1.20)$ & 0.38 & $0.93(0.75-1.14)$ & 0.47 \\
\hline \multirow[t]{4}{*}{$2 q 35$} & $\mathrm{AA}$ & & 1 & & 1 & & 1 & & 1 & \\
\hline & $\mathrm{Aa}$ & & $1.05(0.93-1.19)$ & & $1.04(0.87-1.24)$ & & $1.00(0.81-1.23)$ & & $1.12(0.80-1.58)$ & \\
\hline & aa & & $1.20(1.05-1.37)$ & & 1.09 (0.89-1.33) & & $1.28(1.01-1.61)$ & & $1.38(0.94-2.03)$ & \\
\hline & Per Allele & 0.63 & $1.10(1.03-1.17)$ & 7.47E-03 & $1.04(0.94-1.15)$ & 0.40 & $1.13(1.01-1.27)$ & 0.04 & $1.18(0.97-1.43)$ & 0.09 \\
\hline \multirow[t]{4}{*}{ ESR1 7766585} & $\mathrm{AA}$ & & 1 & & 1 & & 1 & & 1 & \\
\hline & $\mathrm{Aa}$ & & $1.23(1.10-1.37)$ & & $1.29(1.09-1.52)$ & & $1.18(0.97-1.43)$ & & 1.07 (0.79-1.44) & \\
\hline & aа & & $1.02(0.74-1.41)$ & & $0.98(0.60-1.61)$ & & $1.27(0.76-2.14)$ & & $0.51(0.18-1.41)$ & \\
\hline & Per Allele & 0.47 & $1.16(1.05-1.27)$ & $2.66 \mathrm{E}-03$ & $1.19(1.03-1.37)$ & 0.02 & $1.16(0.99-1.37)$ & 0.07 & $0.96(0.74-1.24)$ & 0.75 \\
\hline \multirow[t]{4}{*}{ HCN1 981782} & $\mathrm{AA}$ & & 1 & & 1 & & 1 & & 1 & \\
\hline & $\mathrm{Aa}$ & & $0.97(0.87-1.09)$ & & $1.04(0.87-1.24)$ & & $1.01(0.82-1.23)$ & & $0.87(0.63-1.20)$ & \\
\hline & aа & & $0.86(0.75-0.99)$ & & $0.84(0.68-1.04)$ & & $1.00(0.79-1.28)$ & & $0.87(0.59-1.27)$ & \\
\hline & Per Allele & 0.50 & $0.93(0.87-1.00)$ & 0.04 & $0.92(0.83-1.02)$ & 0.13 & $1.00(0.89-1.13)$ & 0.98 & $0.93(0.77-1.13)$ & 0.46 \\
\hline \multirow[t]{4}{*}{ ESR1 851987} & $\mathrm{AA}$ & & 1 & & 1 & & 1 & & 1 & \\
\hline & $\mathrm{Aa}$ & & $0.91(0.82-1.02)$ & & $0.91(0.78-1.06)$ & & $0.97(0.80-1.17)$ & & $0.92(0.69-1.22)$ & \\
\hline & aа & & $0.88(0.74-1.05)$ & & $0.92(0.71-1.20)$ & & $0.83(0.61-1.13)$ & & $0.76(0.46-1.25)$ & \\
\hline & Per Allele & 0.89 & $0.93(0.86-1.00)$ & 0.06 & $0.94(0.84-1.05)$ & 0.29 & $0.93(0.81-1.07)$ & 0.30 & $0.89(0.72-1.10)$ & 0.28 \\
\hline
\end{tabular}

and one may speculate that rs851987 either participates in this biological effect or is linked to other causal variants.

One SNP (rs13281615 in 8q4) first described by Easton et al. [1] showed a weak tendency for interaction with use of HRT. The minor allele seems to confer increased breast cancer risk in HRT non-users but no excess risk in current users. The association in nonusers is strong with a per-allele OR $(95 \% \mathrm{CI})$ of 1.20 $(1.10-1.31)\left(\mathrm{p}\right.$-trend $\left.=6.1 \times 10^{-5}\right)$ compared to a per-allele OR $(95 \% \mathrm{CI})$ of $1.08(1.01-1.15)(\mathrm{p}$-trend $=0.03)$ in all 
Table 4 OR adjusted for Age and Study Population, stratified by HRT

\begin{tabular}{|c|c|c|c|c|c|c|c|c|c|c|c|c|}
\hline \multirow[t]{3}{*}{ SNP rs nr } & \multirow[t]{3}{*}{ Genotype } & \multirow{3}{*}{$\begin{array}{l}\text { p-value for } \\
\text { interaction }\end{array}$} & \multicolumn{2}{|c|}{ All individuals* } & \multirow{2}{*}{\multicolumn{2}{|c|}{$\begin{array}{c}\text { HRT Non Users* } \\
\text { case/contr: 1536/2949 } \\
\end{array}$}} & \multirow{2}{*}{\multicolumn{2}{|c|}{$\begin{array}{l}\text { HRT Current Users* } \\
\text { case/contr: } 494 / 800\end{array}$}} & \multirow{2}{*}{\multicolumn{2}{|c|}{$\begin{array}{c}\text { HRT Estrogen* } \\
\text { case/contr: } 185 / 324 \\
\end{array}$}} & \multirow{2}{*}{\multicolumn{2}{|c|}{$\begin{array}{c}\text { HRT Comb* } \\
\text { case/contr:298/455 }\end{array}$}} \\
\hline & & & \multicolumn{2}{|c|}{ case/contr: $2869 / 4480$} & & & & & & & & \\
\hline & & & OR $95 \% \mathrm{Cl}$ & $p$-trend & OR 95\%Cl & p-trend & OR 95\%Cl & $\overline{p \text {-trend }}$ & OR $95 \% \mathrm{Cl}$ & $\overline{p \text {-trend }}$ & OR $95 \% \mathrm{Cl}$ & p-trend \\
\hline \multirow[t]{4}{*}{ FGFR2 2981582} & AA & & 1 & & 1 & & 1 & & 1 & & 1 & \\
\hline & $\mathrm{Aa}$ & & $1.23(1.10-1.37)$ & & $1.22(1.06-1.41)$ & & $1.16(0.89-1.50)$ & & $1.15(0.75-1.75)$ & & $1.17(0.83-1.65)$ & \\
\hline & aа & & $1.48(1.29-1.69)$ & & $1.55(1.29-1.89)$ & & $1.63(1.17-2.26)$ & & $1.44(0.86-2.42)$ & & $1.87(1.21-2.89)$ & \\
\hline & Per Allele & 0,83 & $1.22(1.14-1.30)$ & $1.34 \mathrm{E}-08$ & $1.24(1.14-1.36)$ & $2.04 \mathrm{E}-06$ & $1.26(1.07-1.49)$ & 4.83E-03 & $1.20(0.92-1.55)$ & 0.18 & $1.34(1.08-1.66)$ & 7.30E-03 \\
\hline \multirow[t]{4}{*}{ TNRC9 3803662} & AA & & 1 & & 1 & & 1 & & 1 & & 1 & \\
\hline & $\mathrm{Aa}$ & & $1.14(1.03-1.26)$ & & $1.15(1.01-1.31)$ & & $1.17(0.91-1.49)$ & & $1.31(0.89-1.94)$ & & $1.13(0.83-1.56)$ & \\
\hline & aа & & $1.39(1.17-1.67)$ & & $1.48(1.18-1.86)$ & & $1.47(0.96-2.27)$ & & $1.56(0.79-3.11)$ & & $1.53(0.86-2.69)$ & \\
\hline & Per Allele & 0,98 & $1.16(1.08-1.25)$ & $5.34 \mathrm{E}-05$ & $1.19(1.08-1.31)$ & 3.74E-04 & $1.19(1.00-1.43)$ & 0.05 & $1.28(0.96-1.70)$ & 0.09 & $1.19(0.94-1.51)$ & 0.15 \\
\hline \multirow[t]{4}{*}{ MAP3K1 889312} & AA & & 1 & & 1 & & 1 & & 1 & & 1 & \\
\hline & $\mathrm{Aa}$ & & $1.21(1.09-1.34)$ & & $1.27(1.11-1.45)$ & & $1.04(0.82-1.32)$ & & $0.87(0.59-1.29)$ & & $1.13(0.82-1.54)$ & \\
\hline & aа & & $1.29(1.09-1.54)$ & & $1.20(0.95-1.53)$ & & $1.54(1.02-2.33)$ & & $1.81(0.98-3.33)$ & & $1.31(0.74-2.33)$ & \\
\hline & Per Allele & 0,12 & $1.17(1.08-1.26)$ & 4.26E-05 & $1.17(1.06-1.29)$ & $1.90 \mathrm{E}-03$ & 1.15 (0.97-1.38) & 0.11 & $1.16(0.88-1.52)$ & 0.28 & $1.14(0.90-1.44)$ & 0.29 \\
\hline \multirow[t]{4}{*}{$8 q 2413281615$} & AA & & 1 & & 1 & & 1 & & 1 & & 1 & \\
\hline & $\mathrm{Aa}$ & & $1.12(1.00-1.24)$ & & $1.20(1.04-1.39)$ & & $1.05(0.81-1.34)$ & & $0.90(0.60-1.36)$ & & $1.10(0.80-1.53)$ & \\
\hline & aа & & $1.15(1.00-1.32)$ & & $1.43(1.20-1.71)$ & & $0.86(0.61-1.20)$ & & $0.88(0.52-1.51)$ & & $0.84(0.53-1.32)$ & \\
\hline & Per Allele & 0,03 & $1.08(1.01-1.15)$ & 0.03 & $1.20(1.10-1.31)$ & $6.10 \mathrm{E}-05$ & $0.95(0.81-1.12)$ & 0.52 & $0.93(0.72-1.22)$ & 0.61 & $0.95(0.77-1.18)$ & 0.67 \\
\hline \multirow[t]{4}{*}{ LSP1 3817198} & $\mathrm{AA}$ & & 1 & & 1 & & 1 & & 1 & & 1 & \\
\hline & $\mathrm{Aa}$ & & $1.12(1.02-1.24)$ & & $1.13(0.99-1.29)$ & & $1.29(1.01-1.65)$ & & $1.32(0.89-1.94)$ & & $1.30(0.95-1.80)$ & \\
\hline & aа & & $1.19(1.00-1.40)$ & & $1.06(0.85-1.31)$ & & $1.68(1.12-2.53)$ & & $1.19(0.61-2.33)$ & & $2.35(1.37-4.03)$ & \\
\hline & Per Allele & 0,13 & $1.10(1.02-1.18)$ & 8.65E-03 & $1.06(0.97-1.17)$ & 0.21 & $1.30(1.09-1.55)$ & 4.07E-03 & $1.18(0.89-1.56)$ & 0.26 & $1.44(1.14-1.82)$ & $2.16 \mathrm{E}-03$ \\
\hline \multirow[t]{4}{*}{$2 q 35$} & $\mathrm{AA}$ & & 1 & & 1 & & 1 & & 1 & & 1 & \\
\hline & $\mathrm{Aa}$ & & $1.05(0.93-1.19)$ & & $1.05(0.89-1.23)$ & & $0.95(0.72-1.27)$ & & $0.77(0.48-1.25)$ & & $1.13(0.78-1.63)$ & \\
\hline & aa & & $1.20(1.05-1.37)$ & & $1.15(0.97-1.38)$ & & $1.20(0.87-1.67)$ & & $1.26(0.75-2.10)$ & & $1.20(0.78-1.85)$ & \\
\hline & Per Allele & 0,68 & $1.10(1.03-1.17)$ & 7.47E-03 & $1.08(0.98-1.17)$ & 0.11 & $1.10(0.93-1.29)$ & 0.26 & $1.14(0.88-1.48)$ & 0.33 & $1.10(0.88-1.36)$ & 0.40 \\
\hline
\end{tabular}


Table 4 OR adjusted for Age and Study Population, stratified by HRT (Continued)

\begin{tabular}{|c|c|c|c|c|c|c|c|c|c|c|c|c|}
\hline \multirow[t]{4}{*}{ ESR1 7766585} & $\mathrm{AA}$ & & 1 & & 1 & & 1 & & 1 & & 1 & \\
\hline & $\mathrm{Aa}$ & & $1.23(1.10-1.37)$ & & $1.23(1.07-1.43)$ & & $1.05(0.81-1.36)$ & & $1.31(0.85-2.03)$ & & $0.88(0.63-1.23)$ & \\
\hline & aa & & $1.02(0.74-1.41)$ & & $1.01(0.67-1.53)$ & & $1.29(0.61-2.73)$ & & $1.43(0.52-3.93)$ & & $0.86(0.26-2.81)$ & \\
\hline & Per Allele & 0,47 & $1.16(1.05-1.27)$ & 2.66E-03 & $1.15(1.02-1.31)$ & 0.02 & $1.07(0.86-1.34)$ & 0.53 & $1.26(0.89-1.79)$ & 0.19 & $0.89(0.66-1.21)$ & 0.46 \\
\hline \multirow[t]{4}{*}{ HCN1 981782} & $\mathrm{AA}$ & & 1 & & 1 & & 1 & & 1 & & 1 & \\
\hline & $\mathrm{Aa}$ & & $0.97(0.87-1.09)$ & & $0.88(0.76-1.02)$ & & $1.15(0.86-1.53)$ & & $0.88(0.57-1.38)$ & & $1.34(0.92-1.96)$ & \\
\hline & aa & & $0.86(0.75-0.99)$ & & $0.80(0.66-0.95)$ & & $0.93(0.66-1.31)$ & & $0.74(0.43-1.28)$ & & $1.05(0.67-1.64)$ & \\
\hline & Per Allele & 0,26 & $0.93(0.87-1.00)$ & 0.04 & $0.89(0.81-0.98)$ & 0.01 & $0.97(0.82-1.14)$ & 0.70 & $0.86(0.66-1.13)$ & 0.28 & $1.02(0.82-1.27)$ & 0.84 \\
\hline \multirow[t]{4}{*}{ ESR1 851987} & AA & & 1 & & 1 & & 1 & & 1 & & 1 & \\
\hline & $\mathrm{Aa}$ & & $0.91(0.82-1.02)$ & & $0.94(0.82-1.07)$ & & $0.80(0.62-1.04)$ & & $0.94(0.63-1.43)$ & & $0.73(0.52-1.03)$ & \\
\hline & aа & & $0.88(0.74-1.05)$ & & $0.90(0.72-1.13)$ & & $0.93(0.62-1.42)$ & & $0.88(0.46-1.68)$ & & $0.96(0.54-1.69)$ & \\
\hline & Per Allele & 0,52 & $0.93(0.86-1.00)$ & 0.06 & $0.94(0.86-1.04)$ & 0.24 & $0.90(0.75-1.09)$ & 0.28 & $0.94(0.70-1.25)$ & 0.67 & $0.87(0.68-1.12)$ & 0.29 \\
\hline
\end{tabular}


users. The SNP is situated in region $8 \mathrm{q} 24$ that contains no known genes but is in close proximity to FAM84B (coding for a breast cancer membrane associated protein) and the proto-oncogene MYC. The 8q24 locus has previously been reported to associate with other types of cancer in addition to breast cancer [22] and to be more strongly associated with ER + than ER- tumours [23].

Since the first GWAS on breast cancer was published in 2007 several replication and interaction studies of varying sizes have been published [24-27]. In 2010, a large interaction study comprising 7610 breast cancer cases from the Million Women Study in UK was undertaken and potential interactions between 12 different SNPs and 10 different variables (including height, BMI and HRT) were tested [10]. This study did not find (contrary to previous suggestions) any significant geneenvironment interactions. Our study originally included ten of the same polymorphisms as in the Million Women Study (excluding rs1982073 in TGFB1 and rs1800054 in ATM), but also included one additional SNP from Easton et al. [1] and two additional SNPs from our own candidate CpG study [11] (rs7766585 and rs851987 both in ESR1). Although our material is not as large, our study is comprised of three well described study-populations, two of which were prospectively followed for breast cancer incidence using the comprehensive, population-based Swedish Cancer Registry [28]. Thus, our complete case ascertainment and ability to select matched controls from the same study base is likely to have resulted in low risk for selection biases. However, the intervals between data collection, blood sampling, and diagnosis differ substantially between the three different study populations, something that might be considered a limitation of the study.

Considering demographic traits, participants in the MDCS have a slightly higher socioeconomic status than the general population, but as this selection is the same for the study base from which cases and controls are derived, it should not affect the validity of our study [13]. MDCS participants were recruited at age 45-65 years. The exclusion of prevalent cases removes early breast cancer cases from this population. While the NHSDS participants were primarily included from age 40 and upwards, mammography screening had identified some cases as young as 27 years. In Iceland prevalent cases of breast cancer were recruited at varying times after diagnosis, resulting in an exclusion of early lethal cases and older women with other causes of death. As the Icelandic controls were collected later and from the same sample population as the cases there is the possibility of selection bias. Another limitation of our study is the fact that HRT is reported only once (at recruitment) without information about duration. We also lacked information about other risk factors than age, height,
BMI, HRT and therefore could not adjust our results for other potential confounders.

\section{Conclusions}

Our evaluation of genetic predisposition for breast cancer in relation to three different environmental risk factors found no significant gene-environment interactions. We did find tendencies for certain SNPs to exert an effect on breast cancer risk only in women with certain phenotypes. In particular the potential interaction between height and rs851987 in ESR1 in relation to breast cancer risk could merit further investigation. However, independent studies with many more cases would be needed to verify this finding.

\section{Competing interests}

The authors declare that they have no competing interests.

\section{Authors' contributions}

SH had full access to all the data and in the study and takes responsibility for the integrity of the data and the accuracy of the data analysis. SH and JC are the principal investigators of the study and were responsible for the planning of the study. JD participated in the conception and design of the study and contributed with supervising, funding, and administrative support. MILI participated in designing the study. JEE and PL contributed samples to the study. SH and MILI performed all genetic analysis. SH analyzed the data (data extraction and statistical analysis). SH, JC, JD, SB and JM interpreted the data. SH performed the literature search. SH wrote; JC, JD, JM revised; MILI, $\mathrm{SB}, \mathrm{JEE}$, and PL reviewed the paper and contributed suggestions for improvement that were implemented. All authors approved the final version.

\section{Acknowledgements}

We thank Anders Dahlin (MDCS), Åsa Ågren (NSHSD) and Holmfridur Hilmarsdottir (UI/ICS) for sample retrieval and handling, Maria Sterner and Liselott Hall at RSKC (Malmö) research facility for technical assistance and the Icelandic Cancer Registry for providing data. This work was supported by the European Union Network of Excellence grant "Cancer Control using Population-based Registries and Biobanks" and the Breast Cancer Network at Lund University.

\section{Author details}

${ }^{1}$ Department of Medical Microbiology, Lund University, Malmö, Sweden. ${ }^{2}$ Department of Clinical Chemistry, Lund University, Malmö, Sweden. ${ }^{3}$ Department of Surgery, Lund University, Malmö, Sweden. ${ }^{4}$ Region Skånes Biobank, Wallenberg Laboratory, Malmö, Sweden. ${ }^{5}$ Cancer Research Laboratory, Faculty of Medicine, University of Iceland, Reykjavik, Iceland. ${ }^{6}$ Department of Oncology, Norrlands University Hospital, Umeå, Sweden. 7 Labmedicin Skåne, Clinical Chemistry in Lund, Lund, Sweden. ${ }^{8}$ Departments of Laboratory Medicine, Medical Epidemiology \& Biostatistics, Karolinska Institutet and Karolinska Hospital, Stockholm, Sweden.

Received: 13 September 2011 Accepted: 5 June 2012 Published: 22 June 2012

\section{References}

1. Easton DF, Pooley KA, Dunning AM, Pharoah PD, Thompson D, Ballinger DG, Struewing JP, Morrison J, Field H, Luben R, et al: Genome-wide association study identifies novel breast cancer susceptibility loci. Nature 2007, 447:1087-1093.

2. Hunter DJ, Kraft P, Jacobs KB, Cox DG, Yeager M, Hankinson SE, Wacholder S, Wang Z, Welch R, Hutchinson A, et al: A genome-wide association study identifies alleles in FGFR2 associated with risk of sporadic postmenopausal breast cancer. Nat Genet 2007, 39:870-874.

3. Stacey SN, Manolescu A, Sulem P, Rafnar T, Gudmundsson J, Gudjonsson SA, Masson G, Jakobsdottir M, Thorlacius S, Helgason A, et al: Common variants on chromosomes $2 q 35$ and $16 q 12$ confer susceptibility to estrogen receptor-positive breast cancer. Nat Genet 2007, 39:865-869. 
4. Reeves GK, Travis RC, Green J, Bull D, Tipper S, Baker K, Beral V, Peto R, Bell J, Zelenika D, Lathrop M: Incidence of breast cancer and its subtypes in relation to individual and multiple low-penetrance genetic susceptibility loci. JAMA 2010, 304:426-434.

5. Wacholder S, Hartge P, Prentice R, Garcia-Closas M, Feigelson HS, Diver WR, Thun MJ, Cox DG, Hankinson SE, Kraft P, et al: Performance of common genetic variants in breast-cancer risk models. N Engl J Med 2010, 362:986-993.

6. Hunter DJ, Willett WC: Diet, body size, and breast cancer. Epidemio/ Rev 1993, 15:110-132

7. Key TJ, Verkasalo PK, Banks E: Epidemiology of breast cancer. Lancet Oncol 2001, 2:133-140.

8. Prentice RL, Huang Y, Hinds DA, Peters U, Pettinger M, Cox DR, Beilharz E, Chlebowski RT, Rossouw JE, Caan B, Ballinger DG: Variation in the FGFR2 gene and the effects of postmenopausal hormone therapy on invasive breast cancer. Cancer Epidemiol Biomarkers Prev 2009, 18:3079-3085.

9. Rebbeck TR, DeMichele A, Tran TV, Panossian S, Bunin GR, Troxel AB, Strom BL: Hormone-dependent effects of FGFR2 and MAP3K1 in breast cancer susceptibility in a population-based sample of post-menopausa African-American and European-American women. Carcinogenesis 2009, 30:269-274.

10. Travis RC, Reeves GK, Green J, Bull D, Tipper SJ, Baker K, Beral V, Peto R, Bell J, Zelenika D, Lathrop M: Gene-environment interactions in 7610 women with breast cancer: prospective evidence from the Million Women Study. Lancet 2010, 375:2143-2151.

11. Harlid S, Ivarsson MI, Butt S, Hussain S, Grzybowska E, Eyfjord JE, Lenner P, Forsti A, Hemminki K, Manjer J, et al: A candidate CpG SNP approach identifies a breast cancer associated ESR1-SNP. Int J Cancer 2011, 129:1689-1698.

12. Berglund G, Elmstahl S, Janzon L, Larsson SA: The Malmo Diet and Cancer Study. Design and feasibility. J Intern Med 1993, 233:45-51.

13. Manjer J, Carlsson S, Elmstahl S, Gullberg B, Janzon L, Lindstrom M, Mattisson I, Berglund G: The Malmo Diet and Cancer Study: representativity, cancer incidence and mortality in participants and non-participants. Eur J Cancer Prev 2001, 10:489-499.

14. Pukkala E, Andersen A, Berglund G, Gislefoss R, Gudnason V, Hallmans G, Jellum E, Jousilahti $P$, Knekt $P$, Koskela $P$, et al: Nordic biological specimen banks as basis for studies of cancer causes and control-more than 2 million sample donors, 25 million person years and 100,000 prospective cancers. Acta Oncol 2007, 46:286-307.

15. Manjer J, Elmstahl S, Janzon L, Berglund G: Invitation to a populationbased cohort study: differences between subjects recruited using various strategies. Scand J Public Health 2002, 30:103-112.

16. Cox A, Dunning AM, Garcia-Closas M, Balasubramanian S, Reed MW, Pooley KA, Scollen S, Baynes C, Ponder BA, Chanock S, et al: A common coding variant in CASP8 is associated with breast cancer risk. Nat Genet 2007, 39:352-358.

17. Thompson DA, McPherson LA, Carmeci C, deConinck EC, Weigel RJ: Identification of two estrogen receptor transcripts with novel $5^{\prime}$ exons isolated from a MCF7 cDNA library. J Steroid Biochem Mol Biol 1997, 62:143-153.

18. Lambertini E, Penolazzi L, Giordano S, Del Senno L, Piva R: Expression of the human oestrogen receptor-alpha gene is regulated by promoter $\mathrm{F}$ in MG-63 osteoblastic cells. Biochem J 2003, 372:831-839.

19. Penolazzi L, Lambertini E, Giordano S, Sollazzo V, Traina G, del Senno L, Piva R: Methylation analysis of the promoter $F$ of estrogen receptor alpha gene: effects on the level of transcription on human osteoblastic cells. J Steroid Biochem Mol Biol 2004, 91:1-9.

20. Dahlgren A, Lundmark P, Axelsson T, Lind L, Syvanen AC: Association of the estrogen receptor 1 (ESR1) gene with body height in adult males from two Swedish population cohorts. PLoS One 2008, 3:e1807.

21. Emons J, Chagin AS, Malmlof T, Lekman M, Tivesten A, Ohlsson C, Wit JM, Karperien M, Savendahl L: Expression of vascular endothelial growth factor in the growth plate is stimulated by estradiol and increases during pubertal development. J Endocrinol 2010, 205:61-68.

22. Ghoussaini M, Song H, Koessler T, Al Olama AA, Kote-Jarai Z, Driver KE, Pooley KA, Ramus SJ, Kjaer SK, Hogdall E, et al: Multiple loci with different cancer specificities within the 8q24 gene desert. J Natl Cancer Inst 2008, 100:962-966.
23. Garcia-Closas M, Hall P, Nevanlinna H, Pooley K, Morrison J, Richesson DA, Bojesen SE, Nordestgaard BG, Axelsson CK, Arias Jl, et al: Heterogeneity of breast cancer associations with five susceptibility loci by clinical and pathological characteristics. PLoS Genet 2008, 4:e1000054.

24. Antoniou AC, Sinilnikova OM, McGuffog L, Healey S, Nevanlinna H, Heikkinen T, Simard J, Spurdle AB, Beesley J, Chen X, et al: Common variants in LSP1, 2q35 and 8q24 and breast cancer risk for BRCA1 and BRCA2 mutation carriers. Hum Mol Genet 2009, 18:4442-4456.

25. Gorodnova TV, Kuligina E, Yanus GA, Katanugina AS, Abysheva SN, Togo AV, Imyanitov EN: Distribution of FGFR2, TNRC9, MAP3K1, LSP1, and 8q24 alleles in genetically enriched breast cancer patients versus elderly tumor-free women. Cancer Genet Cytogenet 2010, 199:69-72.

26. Hemminki K, Muller-Myhsok B, Lichtner P, Engel C, Chen B, Burwinkel B, Forsti A, Sutter C, Wappenschmidt B, Hellebrand $H$, et al: Low-risk variants FGFR2, TNRC9 and LSP1 in German familial breast cancer patients. Int J Cancer 2010, 126:2858-2862.

27. Huijts PE, Vreeswijk MP, Kroeze-Jansema KH, Jacobi CE, Seynaeve C, Krol-Warmerdam EM, Wijers-Koster PM, Blom JC, Pooley KA, Klijn JG, et al: Clinical correlates of low-risk variants in FGFR2, TNRC9, MAP3K1, LSP1 and 8 q24 in a Dutch cohort of incident breast cancer cases. Breast Cancer Res 2007, 9:R78.

28. Barlow L, Westergren K, Holmberg L, Talback M: The completeness of the Swedish Cancer Register: a sample survey for year 1998. Acta Oncol 2009, 48:27-33.

doi:10.1186/1472-6874-12-17

Cite this article as: Harlid et al:: Interactive effect of genetic susceptibility with height, body mass index, and hormone replacement therapy on the risk of breast cancer. BMC Women's Health 2012 12:17.

\section{Submit your next manuscript to BioMed Central and take full advantage of:}

- Convenient online submission

- Thorough peer review

- No space constraints or color figure charges

- Immediate publication on acceptance

- Inclusion in PubMed, CAS, Scopus and Google Scholar

- Research which is freely available for redistribution 